\title{
Removing fences from sweep motion videos using global 3D reconstruction and fence-aware light field rendering
}

\author{
Chanya Lueangwattana ${ }^{1}$, Shohei Mori ${ }^{2}(\bowtie)$, and Hideo Saito ${ }^{1}$
}

(C) The Author(s) 2018. This article is published with open access at Springerlink.com

\begin{abstract}
Diminishing the appearance of a fence in an image is a challenging research area due to the characteristics of fences (thinness, lack of texture, etc.) and the need for occluded background restoration. In this paper, we describe a fence removal method for an image sequence captured by a user making a sweep motion, in which occluded background is potentially observed. To make use of geometric and appearance information such as consecutive images, we use two well-known approaches: structure from motion and light field rendering. Results using real image sequences show that our method can stably segment fences and preserve background details for various fence and background combinations. A new video without the fence, with frame coherence, can be successfully provided.
\end{abstract}

Keywords video; fence; video repair; diminished reality (DR); structure from motion (SfM); light field rendering (LFR)

\section{Introduction}

Recovering occluded objects in a scene, also known as diminished reality (DR), is a challenging issue that has recently received increasing attention [1]. Visual obstacles can appear to be seen through with a variety techniques such as image-inpainting, which is used to fill in the occluded pixels with ones having similar features. Due to the similarity of goals, we can consider the issue of fence removal in images to be a DR problem. Fence removal requires techniques

1 Department of Science and Technology, Keio University, Japan. E-mail: C. Lueangwattana, chanyal@hvrl.ics.keio.ac.jp; H. Saito, saito@hvrl.ics.keio.ac.jp.

2 Institute of Computer Graphics and Vision, Graz University of Technology, Austria. E-mail: S. Mori, s.mori.jp@ieee.org (西).

Manuscript received: 2018-08-21; accepted: 2018-10-31 to diminish the appearance of a fence in an image to create a fence-free view. Such a technique is useful, for example, when a photographer takes a photograph of a tourist landmark but the scene is occluded by a fence (e.g., for security reasons).

There are two challenging issues in fence removal: fence detection, and background restoration (i.e., restoration of pixels occluded by the fence).

The difficulties in fence detection and segmentation lay in the characteristics of fences; they are typically thin, untextured, etc. Thus, many existing methods of segmenting fence pixels from other pixels are fully manual or semi-automated [2-4], although recent work has introduced fully automated methods [5-11].

To fill in the detected fence pixels, we can use an existing DR method such as image-inpainting to fill in the occluded pixels with appropriate content [7]. We may also use multi-viewpoint images to observe the hidden regions $[2,3]$.

While single shot fence removal, and fence removal using video resources, have been discussed for a decade, to the best of our knowledge, removal of fences in video, which introduces issues of frame coherence, has only a few examples $[8,9]$. Furthermore, the state of the art methods are limited due to reliance on ideal regular patterns of fences [9] and depth variance in the background geometry [8].

To further assess these video related issues, in this research, we implemented several baseline methods for comparison with our algorithm which uses globally consistent data to improve frame coherence in the video results. In this paper, we combine a well-known computer vision method, structure from motion (SfM), with one from graphics, light field rendering (LFR).

Our contributions can be summarized as follows: 
- segmenting foreground and background regions based on depth information from SfM and dense reconstruction;

- recovering occluded background pixels using a modified LFR scheme, starting from an image sequence captured by a sweep camera motion;

- a framework for combining the above approaches for fence removal;

- comparisons of four fence removal methods combining two types of fence detection and background restoration, to validate our proposed method.

\section{Related work}

In this section, we review fence pixel detection and background restoration approaches used in fence removal. Table 1 summarizes the existing work and the proposed method to highlight the differences.

\subsection{Fence detection}

Various researches have proposed methods to detect and remove fences in an image or a video. The pioneering fence removal work by Liu et al. [7] used single image foreground-background segmentation relying on the fact that fences have almost regular patterns. Park et al. [2] improved upon Liu et al.'s method by using online learning for fence pattern detection. Nevertheless, the approach still has problems in detecting fences since actual fences are in reality ideally regularly arranged.

Yamashita et al. [10] detected fences based on differences between flash and non-flash images. Since flash only affects nearby objects, the difference between the two images can provide a fence mask. Khasare et al. [3] and Negi et al. [4] provided semi-automated fence detection algorithms for video frames, requiring the user to identify fence pixels by means of line inputs in an image. Here, all sequential images recorded during a sweep motion are aligned temporo-spatially to detect fence pixels, which appear differently to the background pixels in the space. Zhang et al. [11] used a hyperspectral camera to segment fence pixels in hyperspectral space, using concepts of signal separation. Mu et al. [8] proposed a visual parallax based method of fence removal for videos. Xue et al. [14] implemented a successful method to separate reflections and background based on edge-based pixel motion analysis, using a set of frames captured by a user's sweep motion. Yi et al. [9] segmented fences in a video captured by a smart phone, using graph-cut based on gradient oriented histograms and fence geometry.

As well as color information, depth information allows us to segment fence and non-fence pixels by straightforward depth thresholding, as fences are usually closer to the camera than to the background. Jonna et al. [6] used a Kinect sensor to make a fence mask from the depth data. They also used a stereo image captured by a smart phone to compute a disparity map for the same purpose [5].

Due to their simplicity and generality, we follow the depth segmentation approaches to detect fence pixels in video frames without the need for user input. Instead of a stereo pair, we use all video frames to reconstruct the scene, including the fence, for more robust and reliable depth estimation. Our

Table 1 Approaches used in various fence removal methods. Plain background: image methods. Grey background: video methods

\begin{tabular}{llll}
\hline Method & Input & Fence detection & Background restoration \\
\hline Liu et al. [7] & Single photo & Image segmentation & Image inpainting \\
Park et al. [2] & Sweep motion video & Image segmentation & Multi-view + Single-view inpainting \\
Yamashita et al. [10] & Multi-focus images & Images w/ and w/o a flashlight & Reversed projection blurring model \\
Khasare et al. [3] & Sweep motion video & Image matting & Image \\
Negi et al. [4] & Sweep motion video & Image matting & Markov random field (MRF) \\
Zhang et al. [11] & Hyperspectral images & Signal separation & Discontinuity adaptive MRF \\
Jonna et al. [6] & RGB-D from Kinect & Depth thresholding & Image inpainting with ANNF \\
Jonna et al. [5] & Sweep motion video & Depth thresholding & MRF \\
Zhang et al. [12] & Light field camera image & Manual editing & Optimization in time-spatio image stack \\
Barnes et al. [13] & Image collection & Manual editing & Layerd scene restoration \\
Mu et al. [8] & Sweep motion video & Parallax-aware segmentation & Image completion using LSH \\
Yi et al. [9] & Sweep motion video & Graph-cut + Conditional Random Filed Per frame image inpainting \\
Xue et al. [14] & Sweep motion video & Motion-based foreground extraction & Motion-based background extraction \\
Proposed method & Sweep motion video & Depth thresholding & Image \\
\hline
\end{tabular}


experiments also implemented color-based fence detection as a basis for comparison.

\subsection{Background restoration}

When a single image is used as input for fence removal, patch-based synthesis or image inpainting are the only choices for background restoration [15]. Liu et al. [7] used exemplar-based image inpainting [16] to fill in the occluded pixels with pixels sampled from other regions. Yi et al. [9] also applied an image inpainting approach for background restoration at each frame in a video, since their main focus was to develop a fence detection method. Barnes et al. [13] presented the PatchTable method for fast pixel correspondence searching, using locality-sensitive hashing [17] for speedup. They gave occluding object removal examples as an application.

Instead of using image-inpainting approaches, multi-view images may be used since backgrounds are potentially visible from some perspectives even if occluded in others. Park et al. [2] used multi-view images to fill fence pixels with actual observations from different perspectives in which occluded areas are visible. For the remaining unobserved background, they used symmetry-augmented inpainting, which inpaints pixels with bilaterally symmetric patches. Zhang et al. [11] proposed use of two images for their approximate nearest neighbor fields (ANNF). Similar patches between the fence-masked image and the image itself are found first, and then fence pixels are inpainted with the patch with the highest similarity. The output is then used to re-initialize a new inpainting step, and so on, until all pixels are recovered. Zhang et al. [12] proposed PlenoPatch, an interactive light field editing system for regularly arranged viewpoints based on a scene model of overlapped layers at different depths.

Some fence removal work including that of Jonna et al. [6] and Khasare et al. [3] uses image sequences (i.e., video) and a Markov random field (MRF) model to fuse visible pixels from other frames with fence pixels to be replaced. Negi et al. [4] modified the model as a discontinuity adaptive Markov random field (DAMRF) for edge-preserving fence removal at higher image resolutions.

Fence pixels can also be considered as noise in an image [5, 8, 10]. Jonna et al. [5] gave a fence removal image using a total variation (TV) denoising technique [18]. Yamashita et al. [10] used multi-focus images, pictures imaged at different focal lengths, to determine background pixel colors from an image focused at the background. Mu et al. [8] suppressed fence pixels using a robust temporal median filter. Using optical flow analysis, a significant number of images are stacked temporo-spatially. Fence pixels are suppressed in this space to restore background colors with a median filter.

While, as noted, fence removal for a single image has been well-discussed, fence removal for video has drawn little attention apart from Refs. [8, 9, 14]. In fact, none of these papers showed video fence removal results and therefore it lacks discussion of video visual quality issues (e.g., frame coherence and viewpoint dependence). One of these papers notes that its algorithm can maintain frame coherence only when the background is significantly far away from the fence, and therefore, view-dependent properties in background restoration are out of scope [8]. The other paper is mainly concerned with a fence detection algorithm, and simply uses per frame image inpainting without regard for frame coherence, as the authors note [9].

From this starting point, we modify LFR for fence removal tasks to provide frame-coherent video results with view-dependent properties in the restored backgrounds.

\section{Method}

\subsection{Overview}

Given a video of a scene with a fence (Section 3.2), our algorithm generates a de-fenced video. Figure 1 shows an overview of the pipeline of the proposed method. Like existing approaches, it has two phases: fence detection and background restoration.

In fence detection (Section 3.3), a 3D point cloud of the scene is generated using an SfM method (Section 3.3.1). Based on the scene reconstruction, the point cloud is separated into two clouds of points, those belonging to the fence, and other points (Section 3.3.2). A mesh is created from the points (Section 3.3.3). The fence point cloud is then used to generate mask images for every frame in the recorded video (Section 3.3.2).

In background restoration (Section 3.4), the fence pixels are filled with fence-aware LFR results (Section 3.4.2). This rendering is based on a global 


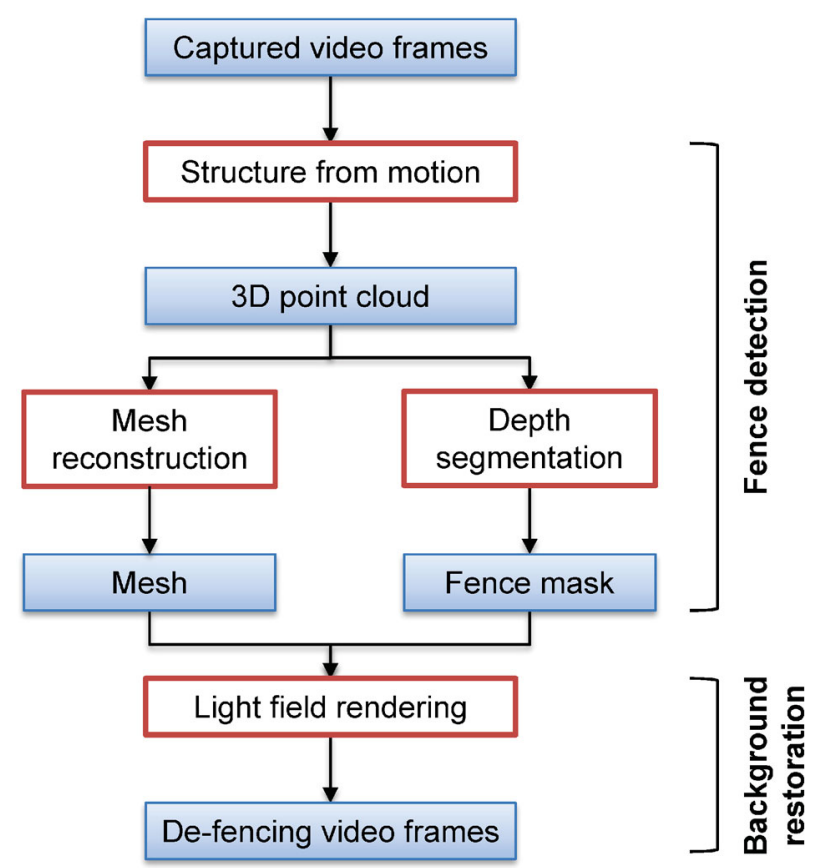

Fig. 1 Overview of proposed method.

reconstruction of the scene, and, therefore the resultant rendering preserves edges, and is framecoherent in the output video.

\subsection{Scene capture}

Following the literature $[2,3]$, we expect that regions occluded by fences in a frame are observable in other frames in a video. To record an image sequence, we have to move the camera in a diagonal direction against the fence rectangle. Note that this diagonal sweep motion is essential for the proposed method to make the camera fully observe the background.

\subsection{Fence detection}

\subsubsection{Scene reconstruction}

Given a video with $N$ frames, geometric information about the captured scene is recovered as a $3 \mathrm{D}$ point cloud by SfM and multi-view stereo [19, 20] using COLMAP, followed by separation of fence and nonfence point clouds. Figures 2(b) and 2(c) show the reconstructed $3 \mathrm{D}$ point cloud corresponding to a scene in Fig. 2(a). The feature points in each frame are detected by SIFT and matched between consecutive frames using sequential matching. Using the obtained point correspondences, each frame is registered with its camera pose and triangulated points as a sparse point cloud. Then, depth and normal maps are computed from registered pairs, and fused to the sparse point cloud to reconstruct a dense
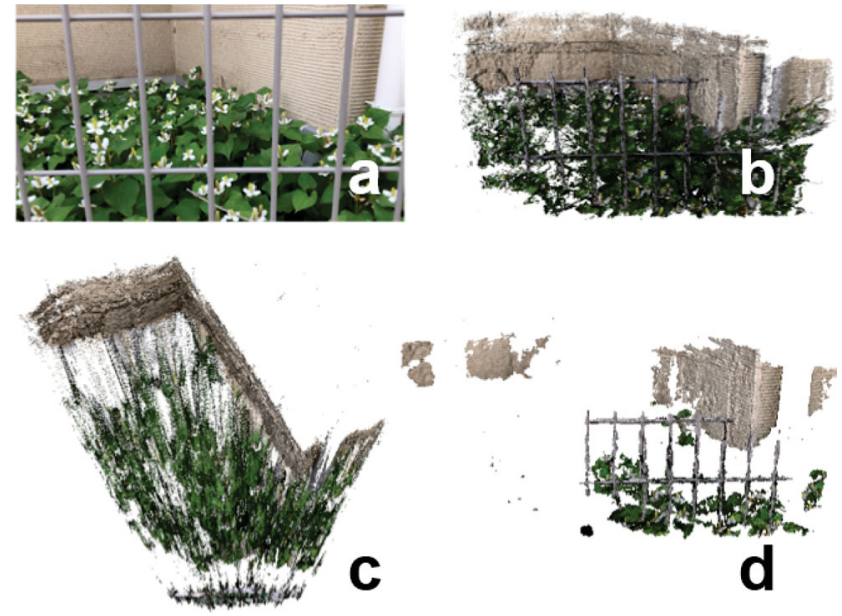

Fig. 2 Scene reconstruction from SfM: (a) a frame from a video sequence, (b) front view of a recovered 3D point cloud with color, (c) top view of the same, (d) 3D background mesh.

point cloud. Note that all frames share the same intrinsic parameters given by bundle adjustment in SfM since the video is captured using a single camera.

\subsubsection{Depth segmentation}

After dense 3D reconstruction, we separate the 3D point cloud into fence $\mathcal{F}$ and non-fence $\overline{\mathcal{F}}$ points. To do so, we select the $T$ closest points (e.g., $T=40 \%$ ) within the $3 \mathrm{D}$ point cloud in a camera coordinate system as a fence point cloud: see Fig. 2(c). Hereafter, we use $\boldsymbol{X}_{\mathcal{F}} \in \mathcal{F}$ and $\boldsymbol{X}_{\overline{\mathcal{F}}}$ denote a fence point and a non-fence point respectively. Consequently, reprojecting $\mathcal{F}$ to data cameras $D_{i}(0 \leqslant i<N)$ results in binary fence mask images $I_{\mathrm{M}}^{D_{i}}$. This re-projection is computed by perspective transformation using the camera parameters and a camera pose extracted from each frame during the SfM phase, using the following equation:

$$
\sigma \tilde{\boldsymbol{x}}=\boldsymbol{A}[\boldsymbol{R} \mid \boldsymbol{t}]_{i} \tilde{\boldsymbol{X}}_{\mathcal{F}}
$$

where $\sigma$ is a scale factor, $\tilde{\boldsymbol{x}}$ gives the homogeneous re-projected point coordinates, $\tilde{\boldsymbol{X}}$ gives homogeneous $3 \mathrm{D}$ point coordinates, $\boldsymbol{A}$ is the $3 \times 3$ matrix of intrinsic parameters, and $[\boldsymbol{R} \mid \boldsymbol{t}]_{i}$ is the $3 \times 4$ matrix of extrinsic parameters for $D_{i}$ describing the camera motion.

As a result, pixels in $I_{\mathrm{M}}^{D_{i}}$ have value 1 if the reprojection $\boldsymbol{x}$ is in the field of view of $D_{i}$, and have vale 0 for other pixels. Figures $3(\mathrm{a})$ and $3(\mathrm{~b})$ show a binary re-projected fence mask, and its appearance in the field of view for some $D_{i}$ respectively.

\subsubsection{Mesh reconstruction}

In COLMAP, the fused 3D point clouds are further triangulated as a dense surface using Poisson surface

\section{(10) TNIVERSTY PRESS}



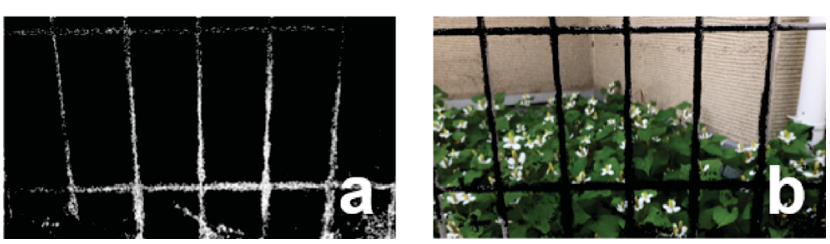

Fig. 3 Fence mask from depth segmentation: (a) a binary mask, (b) fence mask re-projection in the view of data camera $D_{i}$.

reconstruction [21] or Delaunay reconstruction. Figure 2(d) shows the reconstructed surface result for the 3D point cloud in Fig. 2(b); the visible region in the scene is meshed.

The dense surfaces and point cloud are combined as acquired 3D background information $\overline{\mathcal{F}}$, which can densify the depth information when $\overline{\mathcal{F}}$ is projected, and can preserve frame coherence in a video. Figures 4(a) and 4(b) show the differences in background projection without and with use of the mesh respectively.

However, we leave $\mathcal{F}$ as points since fences are thin and can be smoothed out. Instead, we dilate $I_{\mathrm{M}}^{D_{i}}$ to densify the masked regions.

\subsection{Background restoration}

\subsubsection{LFR parameterization}

We now recover the missing pixels in the detected fence regions, i.e., those for which $I_{\mathrm{M}}^{D_{i}}(\boldsymbol{x})$ is 1 , based on LFR, which is an image-based rendering method for generating new views from arbitrary camera positions [22-25]. LFR uses four parameters, $r=(u, v, s, t)$, to represent a scene. As shown in Fig. 5, a ray $\boldsymbol{r}$ represents a light ray that passes through a camera plane at $(u, v)$ and a focal plane at $(s, t)$ in a virtual image $C$. A pixel color at $(s, t)$ in the visual view $C$ can, therefore, be calculated by blending the corresponding colors in data cameras' images $D_{i}$.

\subsubsection{Fence-aware LFR}

To make use of the data obtained so far, we modified LFR [22, 23] as described in the pseudo code in Algorithm 1. As noted in several papers, LFR can
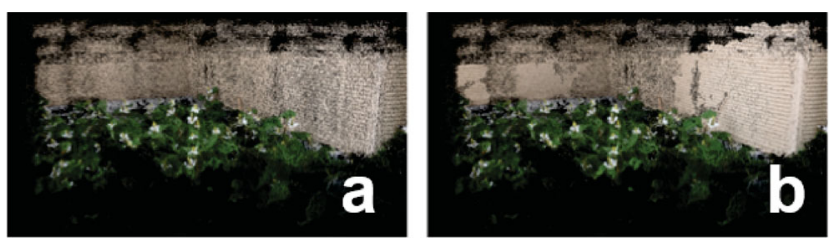

Fig. 4 Background projection from: (a) 3D point cloud, (b) 3D point cloud and mesh.

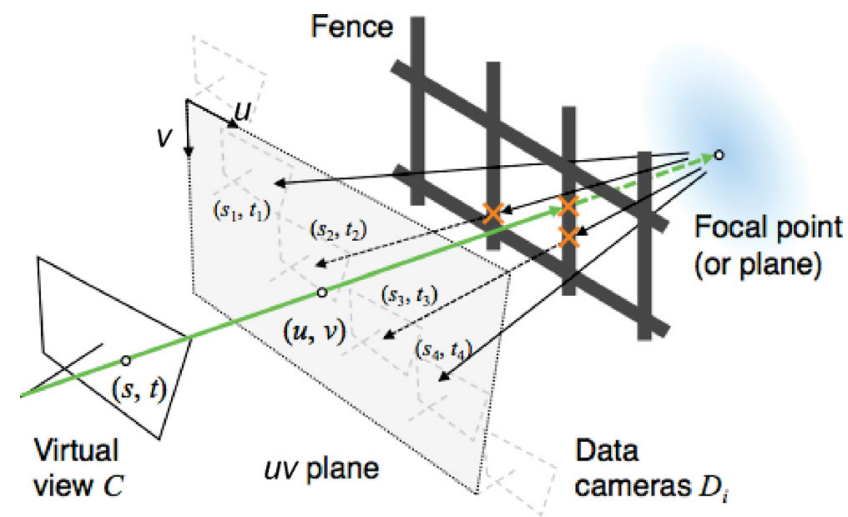

Fig. 5 Light field rendering parameterization.

be improved by using implicit depth from a focal plane $[22,23,25]$ or explicit depth from scene geometry $[26,27]$. We thus implemented explicit depth-based LFR to preserve view-dependent properties in the output scene.

Given a background $3 \mathrm{D}$ point $\boldsymbol{X}_{\overline{\mathcal{F}}}$ at a missing pixel position $\boldsymbol{x}$, and registered data cameras $D_{i}$, we render the background by blending the $D_{i}$ images. The pixels to be blended in $D_{i}$ are calculated by projecting the 3D point to $D_{i}$ as described in Eq. (1). However, masked pixels in $D_{i}$ are given zero weight during blending and, as a result, the fence pixels are not incorporated in the blending results. Furthermore,

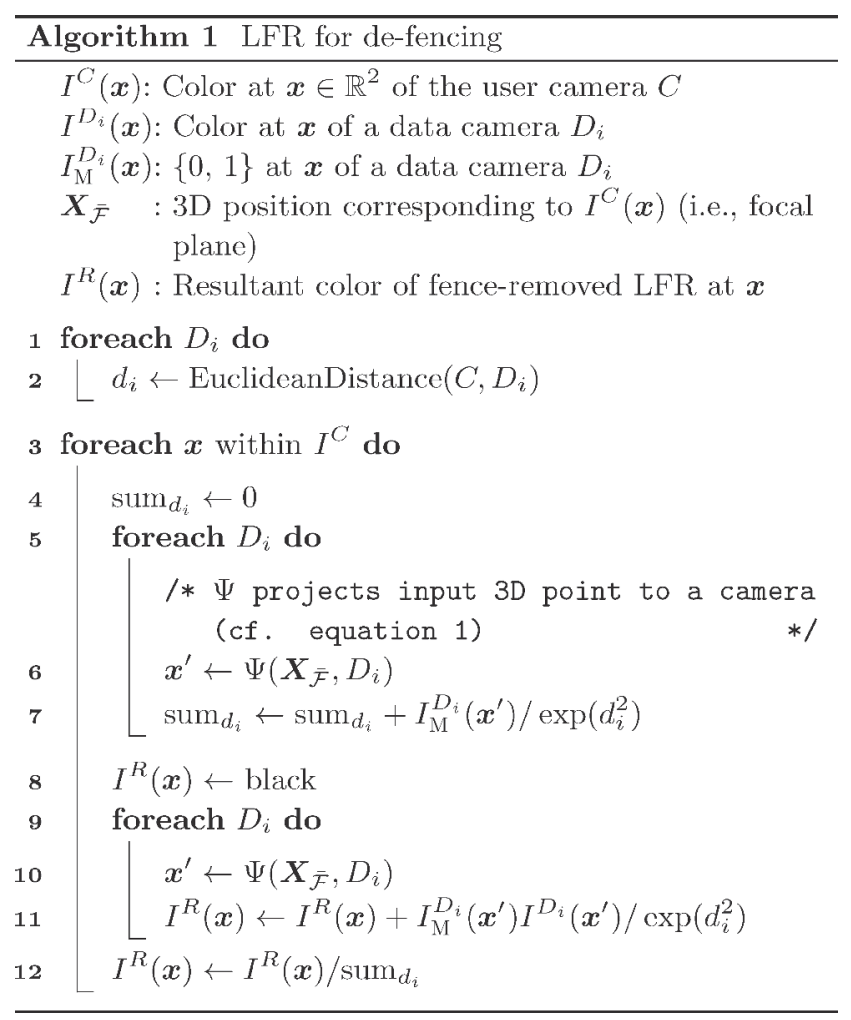


the blending function is weighted inversely by the Euclidean distance between $(u, v)$ 's camera position and the render frame position, giving more weight to a ray from the camera that is more closely aligned with $C$. Figure 6 shows the blending result for the missing pixels masked in Fig. 3(b).

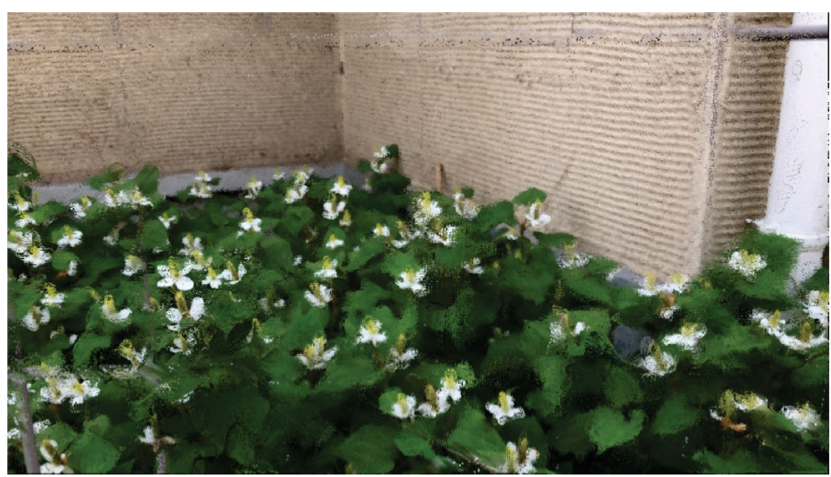

Fig. 6 Blending results using fence-aware LFR for camera data $D_{i}$.

\section{Evaluation}

In this section, we compare the proposed method with three other alternative implementations to discuss their performance in the frame coherence and the visual quality in seven different scenes.

\subsection{Methods for comparison}

Since implementations of published video fence removal methods $[8,9]$ are not publicly available, instead, we implemented four video fence removal methods in combination with two fence detection and two background restorations for comprehensive tests.

For fence segmentation, we implemented a method based on optical flow [28] with $k$-means $(k=2)$ clustering as a baseline. Optical flow techniques have been used in image fence removal work with video resources $[5,6,8,9]$ to estimate pixel motion between consecutive frames. Since fences are normally closer to the camera than the background, fence pixels move more than background pixels during camera translation. Therefore, we can segment pixels into two clusters. We refer to this implementation as "optical flow + binary clustering $(\mathrm{OF}+\mathrm{BC})$ ", while we denote our global depth thresholding "DT".

For background restoration, image-inpainting is one of the major approaches used in fence removal $[2,7,9,11]$ and, therefore, we chose this as a baseline here. Given the fence mask from $\mathrm{OF}+\mathrm{BC}$ or $\mathrm{DT}$, we either apply our fence-aware LFR (FA-LFR) or frame-by-frame PhotoShop Content-Aware Fill (i.e., image-inpainting) (IP); it is an exemplar-based imageinpainting based on PatchMatch [29].

These implementations were combined to provide four fence removal methods: $\mathrm{OF}+\mathrm{BC} / \mathrm{IP}$, $\mathrm{OF}+\mathrm{BC} / \mathrm{FA}-\mathrm{LFR}, \mathrm{DT} / \mathrm{IP}$, and DT/FA-LFR (our proposed method).

\subsection{Dataset}

We recorded seven scenes with combinations of various types of background and fences including challenging irregular ones to confirm the robustness of the proposed method in the face of real-life scene variations. Figure 7 shows stills from the seven scenes along with corresponding fence detection binary masks from $\mathrm{OF}+\mathrm{BC}$ and $\mathrm{DT}$ respectively. The image sequences in each scene were recorded using an iPhone 8 Plus or an iPhone $6 \mathrm{~s}(960 \times 540$ pixels at $30 \mathrm{~Hz})$. The clips in Fig. 7 contained 79 and 437 frames; 10-20 closest (in Euclidean distance) data cameras were used for FA-LFR, depending upon the scene. Table 2 summarizes the setups. As in other video fence removal literature $[8,9,14]$, we always assume

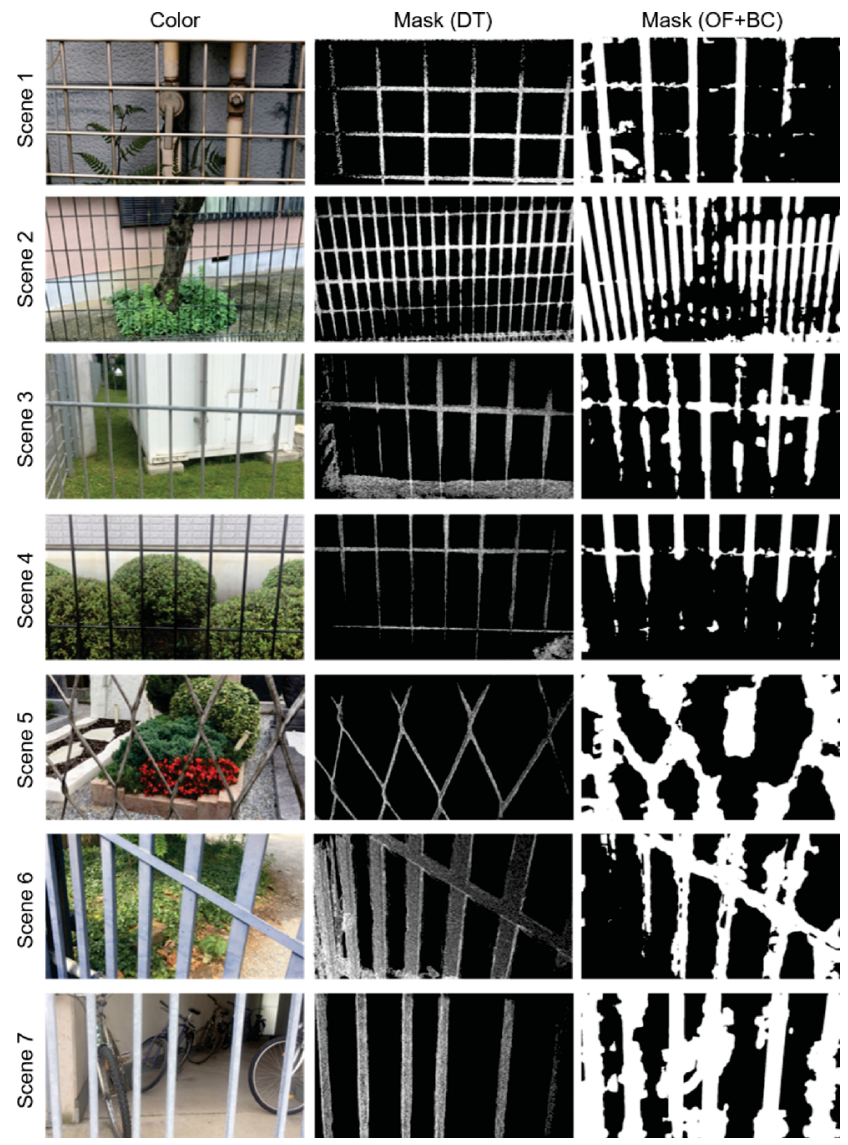

Fig. 7 Fence detection in experimental scenes.

\section{(19) TSIVERSITY PRESS}


Table 2 Experimental scenes

\begin{tabular}{lllcll}
\hline & Fence type & Background & Frames & $T(\%)$ & $N$ \\
\hline 1 & Regular square grid & Wall + pipes & 220 & 25 & 10 \\
2 & Regular rectangle grid & Wall + tree & 437 & 30 & 10 \\
3 & Regular rectangle grid & Grass + warehouse & 116 & 40 & 10 \\
4 & Regular rectangle grid & Wall + shrubbery & 186 & 20 & 20 \\
5 & Distorted rhombus grid & Grave & 87 & 15 & 10 \\
6 & Irregular grid & Grass + ground & 79 & 50 & 10 \\
7 & Irregular lattice & Bicycles & 108 & 30 & 10 \\
\hline
\end{tabular}

the fences to be closer to the user than the background. The fence removal results are best seen in color, in the video in the Electronic Supplementary Material (ESM).

\subsection{Fence removal results and discussion}

Figures $8-11$ show results in three consecutive frames in four scenes to confirm frame coherence. Figures 12-14 show results using OF+BC/IP, DT/IP, $\mathrm{OF}+\mathrm{BC} / \mathrm{FA}-\mathrm{LFR}$, and DT/FA-LFR to highlight differences in the visual quality and frame coherence. In the paper, we only shows comparative results for Scenes 5-7, which include challenging irregular fences; supplemental material in the ESM shows all the results in pictures and video. The areas in the red squares are enlarged on the right of each image to show recovered pixels in detail. In most our experimental scenes, the DT masks more comprehensively mask the fences than $\mathrm{OF}+\mathrm{BC}$ does. An examination of the visual quality and stability shows that our proposed FA-LFR method is more effective in restoring the missing background than IP, either for DT or OF+BC masking, throughout each entire scene.

FA-LFR replaces fence pixels with background pixels by blending view-dependent pixels selected by a global 3D point cloud projection. Pixel color blending based on actual visible backgrounds keeps consistency between input pixels and synthesized ones, while the global 3D point projection keeps frame coherence. These features provide good restoration of object edges (see the pipes in Figs. 8 and 10, rifts between two different materials in Figs. 9 and 11), even in challenging scenes (see Fig. 12) and for unusual object shapes (see the grass in Fig. 9, the shrubs in Fig. 11, and the bicycles in Fig. 14).

On the other hand, IP tends to lose straight lines in
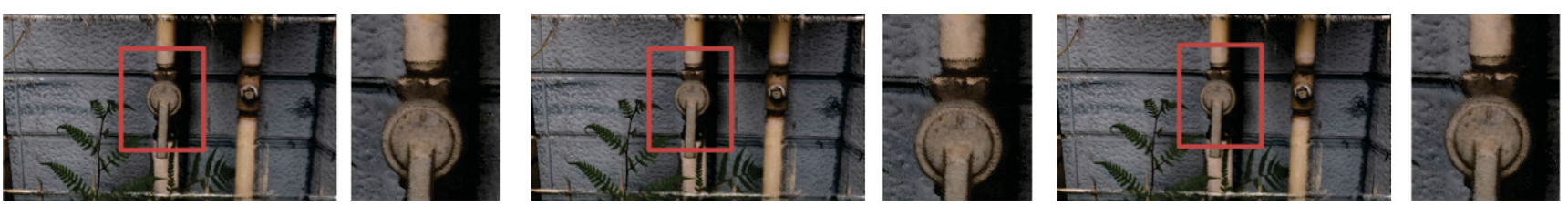

Fig. 8 Background restoration for consecutive frames of Scene 1.
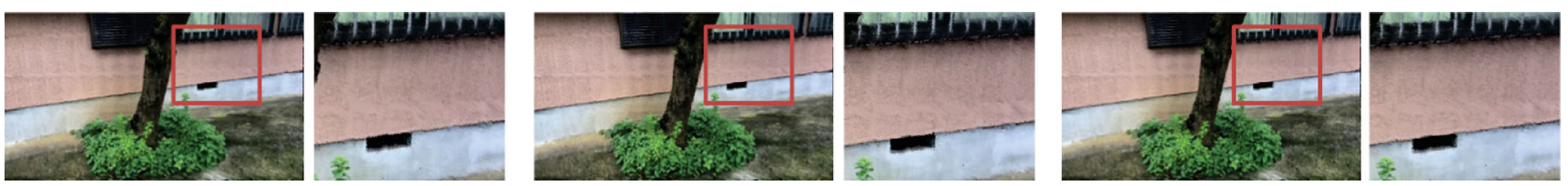

Fig. 9 Background restoration for consecutive frames of Scene 2.
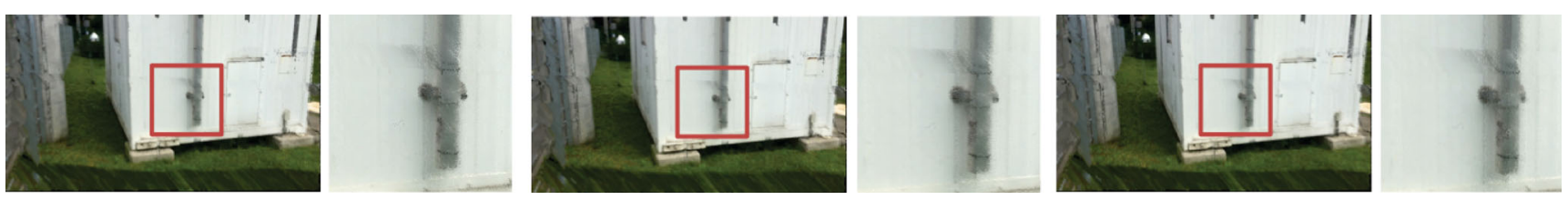

Fig. 10 Background restoration for consecutive frames of Scene 3.
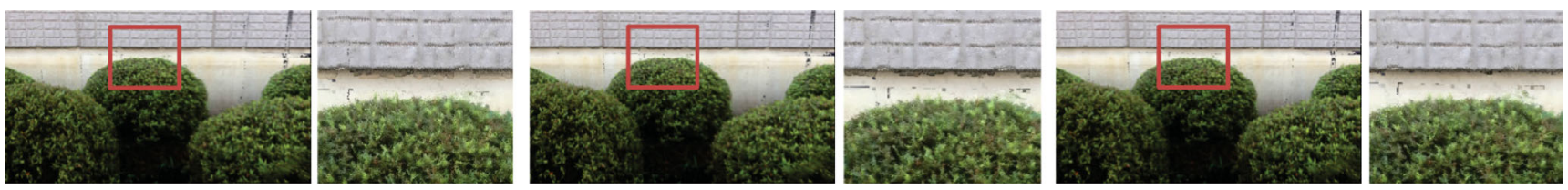

Fig. 11 Background restoration for consecutive frames of Scene 4. 


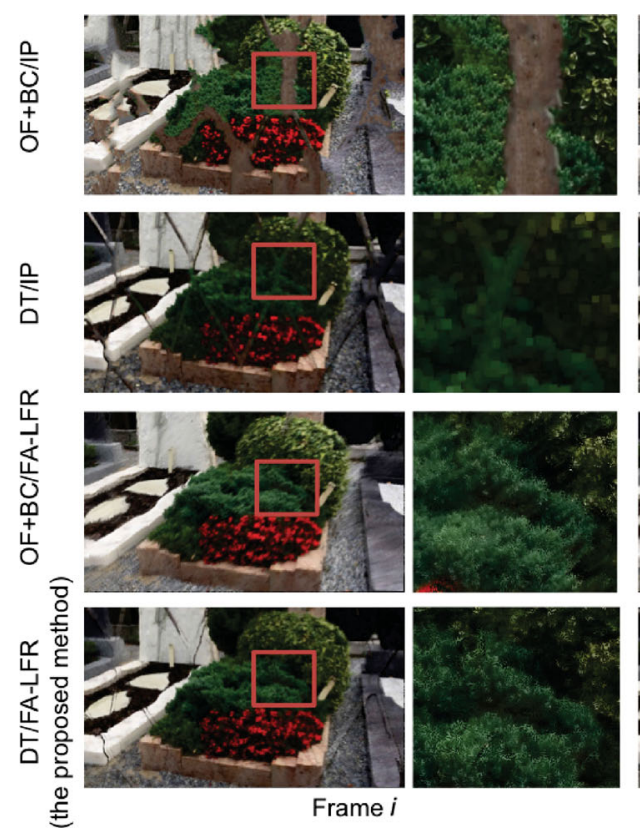

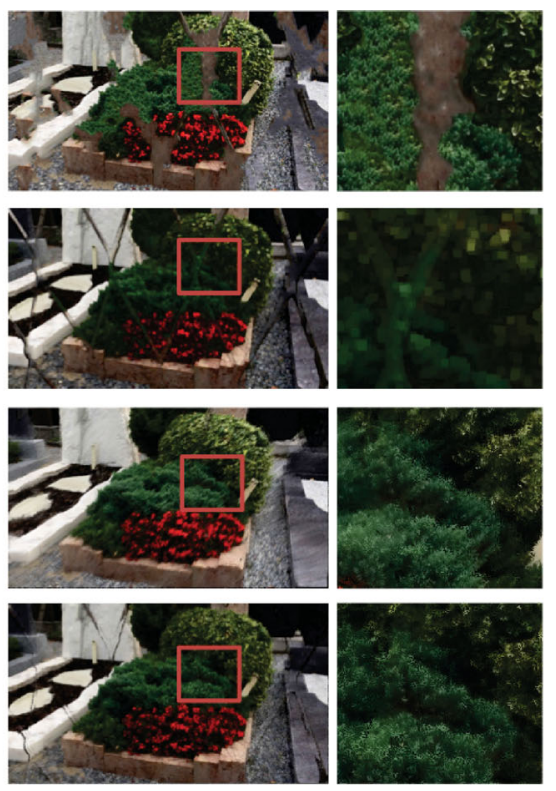

Frame $i+1$
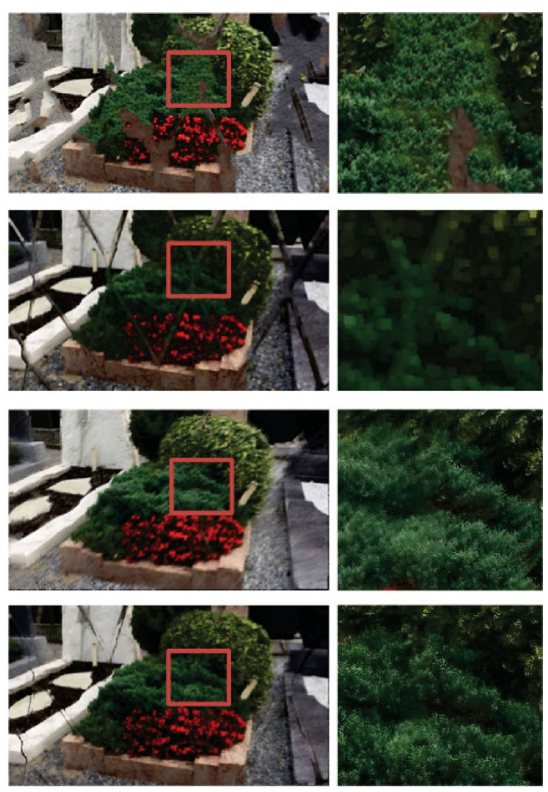

Frame $i+2$

Fig. 12 Comparison of background restoration for consecutive frames of Scene 5
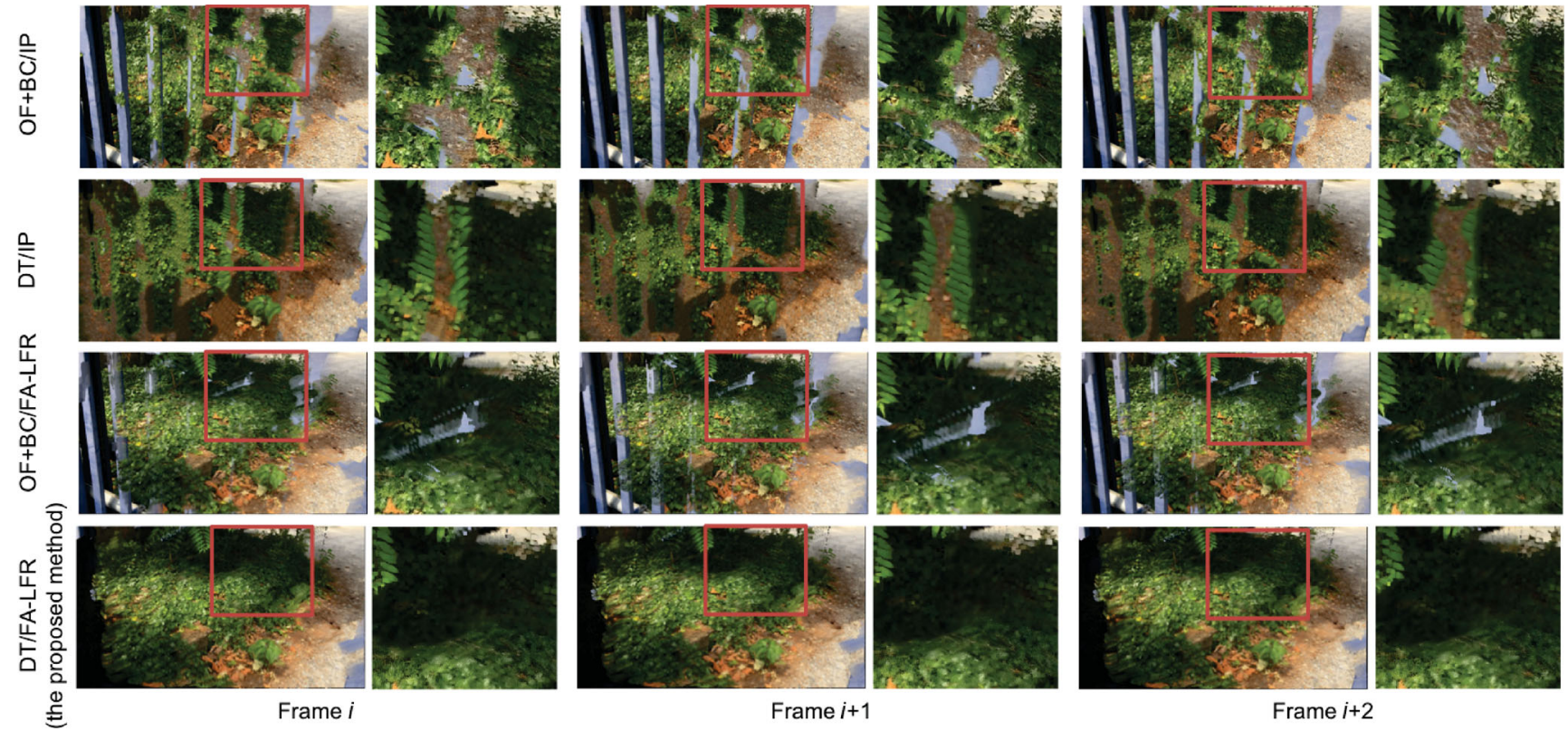

Frame $i+1$

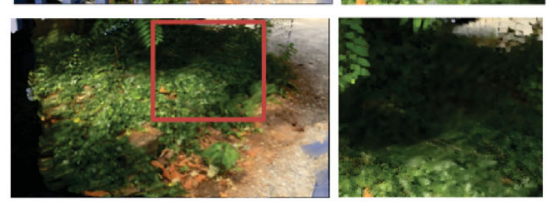

Fig. 13 Comparison of background restoration for consecutive frames of Scene 6

the background when such lines are partially occluded by the fence; in the worst case, IP removes the entire object from the background (see Fig. 14). When fence pixels partially remain after $\mathrm{OF}+\mathrm{BC}$ masking, IP may even recover the fence itself since IP can find clues of the fence in the input image (see Figs. 12 and 13). Such details are normally preserved in the restoration result from FA-LFR.

IP suffers from significant frame by frame changes in appearance of the background due to its random initialization, and frame-incoherent fence detection by $\mathrm{OF}+\mathrm{BC}$. Even with diagonal camera motion, $\mathrm{OF}+\mathrm{BC}$ usually detects only one direction of fence pattern and misses the other (see the binary masks of scene 1, 2, 4, and 5 in Fig. 7). This causes the fence to remain in the restoration results, even sometimes entirely appearing in some frames (see Fig. 12). On the other hand, the DT method provides accurate

\section{(19) TNIVERSITY PRESS}



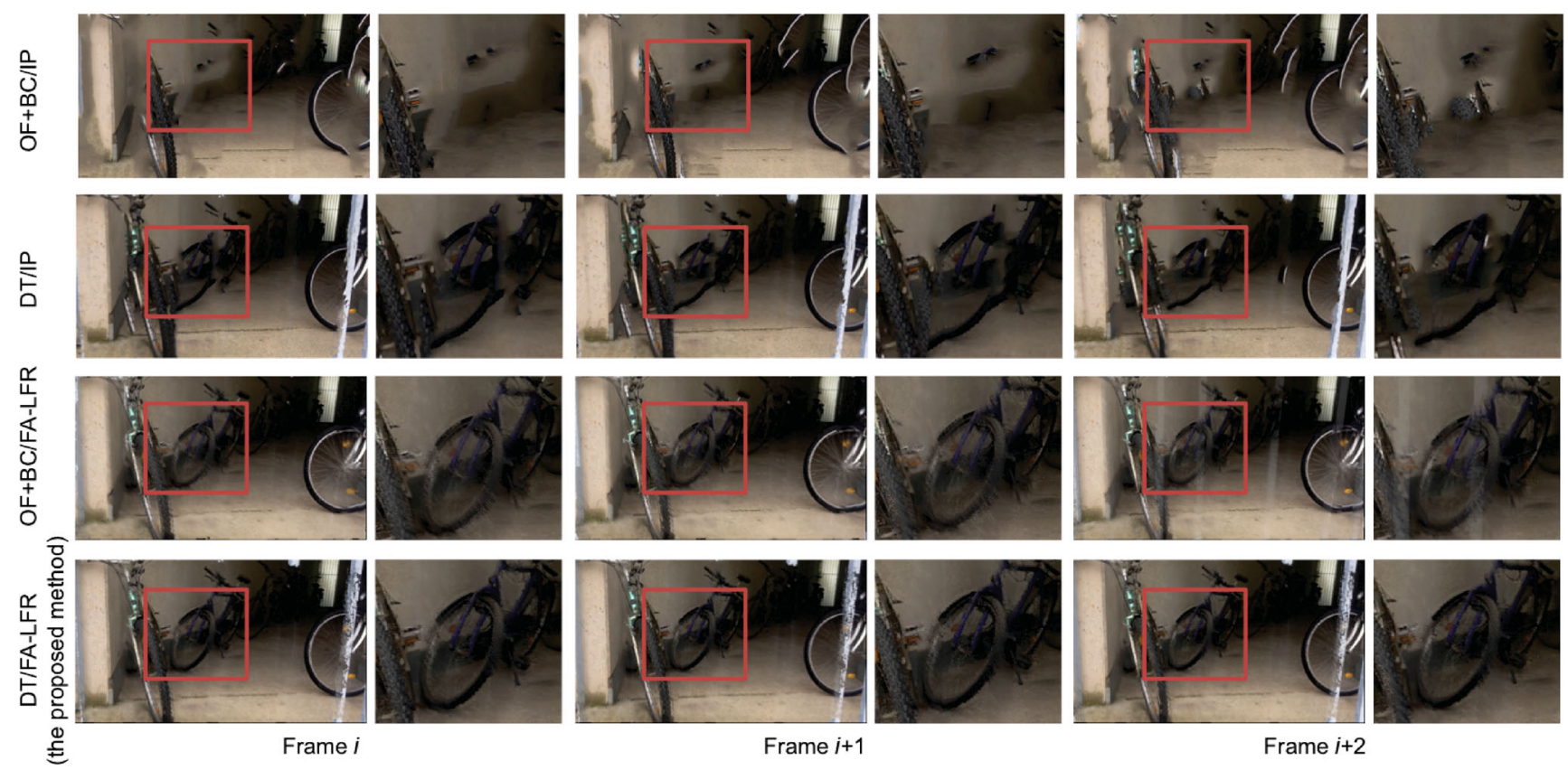

Frame $i+2$

Fig. 14 Comparison of background restoration for consecutive frames of Scene 7 .

and stable fence segmentation across frames due to use of global 3D reconstruction. By comparing fence masking from the two methods using FA-LFR with both, it can be seen that restoration using masking from DT produces better fence removal result: precise fence segmentation obviously leads to the better background restoration quality. If fence pixels still remain after the fence detection step, IP tends to recover the fence itself from the remaining fence pixels (see the DT/IP results in Fig. 12). The proposed method recovers only the background pixels because it takes background depths into account.

While in the most cases, DT/FA-LFR achieved the most plausible results amongst all four methods, we observed failures in some frames. For example, in scenes such as Fig. 15, the algorithm had difficulties in differentiating the fence and the background since the fence's colors are very similar to those in the background, and the backgrounds are close to the fence. The algorithm could successfully detect the horizontal fence, although mis-detects most of the vertical fence close to the tree behind. Nevertheless, the proposed method still provides acceptable results, although the fence is not removed completely.

The DT/FA-LFR approach requires global 3D reconstruction of target scenes. Therefore, for frames of the beginning and the end of the sequence, the algorithm can fail to remove the fence due to a lack of image resources for both 3D reconstruction and LFR, regardless of the accuracy of fence detection. Consequently, artifacts such as black pixels and duplicated colors may be observed. In addition, pixels with no depth information are considered to have infinite depth. Therefore, such pixels may seem blurred, although they are trivial and barely seen in the results.

From the evaluations between the proposed method and the other three implementations, the inconsistent fence detection or the coherent background restoration are major issues for the video fence removal quality. Regarding these issues, the DT/ FA-LFR performed more stable fence detection than

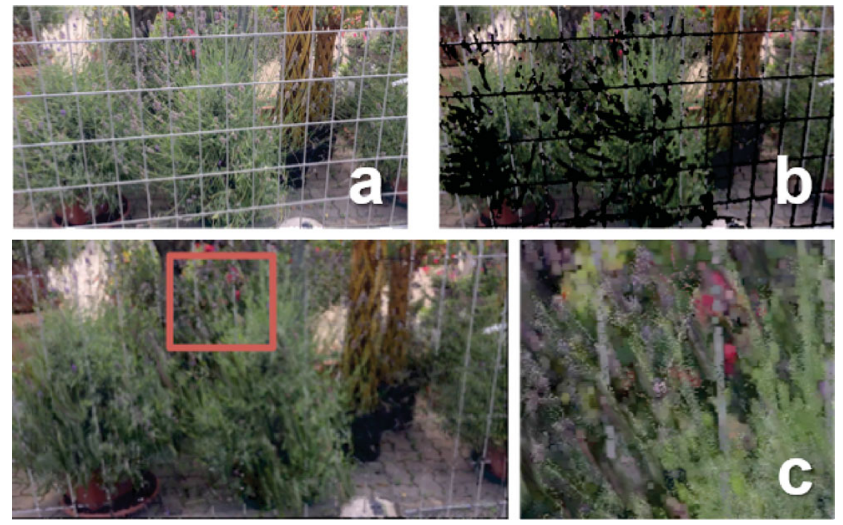

Fig. 15 Failure in DT fence segmentation: (a) input frame, (b) fence mask superimposed on camera data, and (c) result of our method. 
$\mathrm{OF}+\mathrm{BC}$ does and better background pixel recovery than IP does.

A final issue in fence removal methods, including ours, is usability. These methods require users to appropriately move their cameras to sufficiently observe hidden background or distinguish foreground from background $[2,8,9,14]$. User prompting may be helpful. We have found that circular motions give us better results. Many papers suggest to use straight swiping motions to provide significant differences between foreground and background motion in the video frames. Using such approaches, circular motion is not preferable since circular motion always includes different movements. On the other hand, our method uses a conventional SfM approach to obtain camera poses leading to our LFR scheme. As a result, users may move their cameras more carelessly, and strictly straight motions are nor required, but at the same time, users need to move their cameras relatively slowly so as not to lose correspondences between frames. An acceptable speed depends on the distance from the fence to the user.

\section{Conclusions}

This paper has presented an automated method for fence removal from video, using a framework of a combination of SfM-based fence detection and LFR-based background restoration. Allowing use of existing libraries for SfM and LFR, our method has advantages of simplicity and preserving view-dependent appearance in the video result. A qualitative evaluation shows that fences are effectively segmented by depth thresholding in the globally reconstructed space for most experimental scenes. Missing pixels are reliably recovered and the details are preserved using the fence-aware LFR. The final output is a video sequence which better preserves frame coherence than the other three comparative methods.

The proposed method relies on the $3 \mathrm{D}$ point cloud from SfM for both fence segmentation and background restoration. As a result, the user's motion during video recording is important. Appropriate user guidance using augmented reality visualization will solve this issue but remains for future work.

\section{Acknowledgements}

This work was supported in part by Grant-in-Aid from the Japan Society for the Promotion of Science, following Grant No. 16J05114.

Electronic Supplementary Material Supplementary material is available in the online version of this article at https://doi.org/10.1007/s41095-018-0126-8.

\section{References}

[1] Mori, S.; Ikeda, S.; Saito, H. A survey of diminished reality: Techniques for visually concealing, eliminating, and seeing through real objects. IPSJ Transactions on Computer Vision and Applications Vol. 9, 17, 2017.

[2] Park, M.; Brocklehurst, K.; Collins, R. T.; Liu, Y. Image de-fencing revisited. In: Computer Vision - ACCV 2010. Lecture Notes in Computer Science, Vol. 6495. Kimmel, R.; Klette, R.; Sugimoto, A. Eds. Springer Berlin Heidelberg, 422-434, 2011.

[3] Khasare, V. S.; Sahay, R. R.; Kankanhalli, M. S. Seeing through the fence: Image de-fencing using a video sequence. In: Proceedings of the IEEE International Conference on Image Processing, 1351-1355, 2013.

[4] Negi, C. S.; Mandal, K.; Sahay, R. R.; Kankanhalli, M. S. Super-resolution de-fencing: Simultaneous fence removal and high-resolution image recovery using videos. In: Proceedings of the IEEE International Conference on Multimedia and Expo Workshops, 1-6, 2014.

[5] Jonna, S.; Satapathy, S.; Sahay, R. R. Stereo image de-fencing using smartphones. In: Proceedings of the IEEE International Conference on Acoustics, Speech and Signal Processing, 1792-1796, 2017.

[6] Jonna, S.; Voleti, V. S.; Sahay, R. R.; Kankanhalli, M. S. A multimodal approach for image de-fencing and depth inpainting. In: Proceedings of the 8th International Conference on Advances in Pattern Recognition, 1-6, 2015.

[7] Liu, Y.; Belkina, T.; Hays, J. H.; Lublinerman, R. Image de-fencing. In: Proceedings of the IEEE Conference on Computer Vision and Pattern Recognition, 1-8, 2008.

[8] Mu, Y.; Liu, W.; Yan, S. Video de-fencing. IEEE Transactions on Circuits and Systems for Video Technology Vol. 24, No. 7, 1111-1121, 2014.

[9] Yi, R.; Wang, J.; Tan, P. Automatic fence segmentation in videos of dynamic scenes. In: Proceedings of the IEEE Conference on Computer Vision and Pattern Recognition, 705-713, 2016.

[10] Yamashita, A.; Matsui, A.; Kaneko, T. Fence removal from multi-focus images. In: Proceedings of the 20th International Conference on Pattern Recognition, 4532$4535,2010$.

[11] Zhang, Q.; Yuan, Y.; Lu, X. Image de-fencing with hyperspectral camera. In: Proceedings of the International Conference on Computer, Information and Telecommunication Systems, 1-5, 2016.

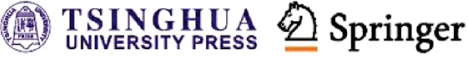


[12] Zhang, F.-L.; Wang, J.; Shechtman, E.; Zhou, Z.Y.; Shi, J.-X.; Hu, S.-M. PlenoPatch: Patch-based plenoptic image manipulation. IEEE Transactions on Visualization and Computer Graphics Vol. 23, No. 5, 1561-1573, 2017.

[13] Barnes, C.; Zhang, F.-L.; Lou, L.; Wu, X.; Hu, S.-M. PatchTable: Efficient patch queries for large datasets and applications. ACM Transactions on Graphics Vol. 34, No. 4, Article No. 97, 2015.

[14] Xue, T.; Rubinstein, M.; Liu, C.; Freeman, W. T. A computational approach for obstruction-free photography. ACM Transactions on Graphics Vol. 34, No. 4, Article No. 79, 2015.

[15] Barnes, C.; Zhang, F.-L. A survey of the state-of-the-art in patch-based synthesis. Computational Visual Media Vol. 3, No. 1, 3-20, 2017.

[16] Criminisi, A.; Prez, P.; Toyama, K. Region filling and object removal by exemplar-based inpainting. IEEE Transactions on Image Processing Vol. 13, No. 9, 12001212, 2004.

[17] Datar, M.; Immorlica, N.; Indyk, P.; Mirrokni, V. S. Locality-sensitive hashing scheme based on p-stable distributions. In: Proceedings of the 20th Annual Symposium on Computational Geometry, 253-262, 2004.

[18] Goldstein, T.; Osher, S. The split Bregman method for L1-regularized problems. SIAM Journal on Imaging Sciences Vol. 2, No. 2, 323-343, 2009.

[19] Schönberger, J. L.; Frahm, J.-M. Structure-from-motion revisited. In: Proceedings of the IEEE Conference on Computer Vision and Pattern Recognition, 4104-4113, 2016.

[20] Schönberger, J. L.; Zheng, E.; Frahm, J.-M.; Pollefeys, M. Pixelwise view selection for unstructured multi-view stereo. In: Computer Vision - ECCV 2016. Lecture Notes in Computer Science, Vol. 990\%. Leibe, B.; Matas, J.; Sebe, N.; Welling, M. Eds. Springer Cham, 501-518, 2016.

[21] Kazhdan, M.; Hoppe, H. Screened poisson surface reconstruction. ACM Transactions on Graphics Vol. 32, No. 3, Article No. 29, 2013.

[22] Davis, A.; Levoy, M.; Durand, F. Unstructured light fields. Computer Graphics Forum Vol. 31, No. 2pt1, 305-314, 2012.

[23] Isaksen, A.; McMillan, L.; Gortler, S. J. Dynamically reparameterized light fields. In: Proceedings of the 27th Annual Conference on Computer Graphics and Interactive Techniques, 297-306, 2000.
[24] Kusumoto, N.; Hiura, S.; Sato, K. Uncalibrated synthetic aperture for defocus control. In: Proceedings of the IEEE Conference on Computer Vision and Pattern Recognition, 2552-2559, 2009.

[25] Levoy, M.; Hanrahan, P. Light field rendering. In: Proceedings of the 23rd Annual Conference on Computer Graphics and Interactive Techniques, 31-42, 1996.

[26] Gortler, S. J.; Grzeszczuk, R.; Szeliski, R.; Cohen, M. F. The lumigraph. In: Proceedings of the 23rd Annual Conference on Computer Graphics and Interactive Techniques, 43-54, 1996.

[27] Buehler, C.; Bosse, M.; McMillan, L.; Gortler, S.; Cohen, M. Unstructured lumigraph rendering. In: Proceedings of the 28th Annual Conference on Computer Graphics and Interactive Techniques, 425432, 2001.

[28] Farnebäck, G. Two-frame motion estimation based on polynomial expansion. In: Image Analysis. Lecture Notes in Computer Science, Vol. 2749. Bigun, J.; Gustavsson, T. Eds. Springer Berlin Heidelberg, 363$370,2003$.

[29] Barnes, C.; Shechtman, E.; Finkelstein, A.; Goldman, D. B. PatchMatch: A randomized correspondence algorithm for structural image editing. ACM Transactions on Graphics Vol. 28, No. 3, Article No. 24, 2009.

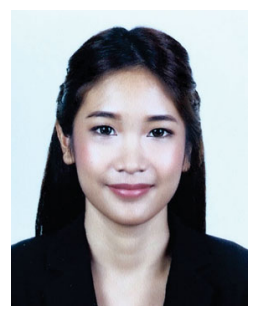

Chanya Lueangwattana received her B.E. degree in electronics and communication engineering from Thammasat University, Thailand, in 2015. Since 2016, she was a master student in the Graduate School of Science and Technology at Keio University, from which she received her M.S. degree in 2018. Her research interests include image processing, computer vision, and diminished reality.

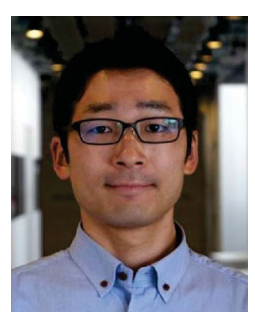

Shohei Mori received his B.S., M.S., and Ph.D. degrees in engineering from Ritsumeikan University, Japan, in 2011, 2013, and 2016, respectively. He was a research fellowship for Young Scientists DC-1 and PD from the Japan Society for the Promotion of Science until 2016 and 2018 respectively. He is currently a university project assistant at Graz University of Technology, Austria. His research interests include diminished reality and related computer vision technology. 


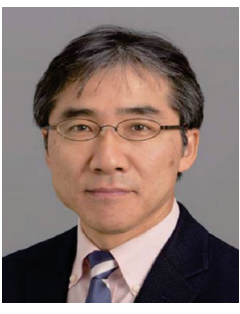

Hideo Saito received his Ph.D. degree in electrical engineering from Keio University, Japan, in 1992. Since then, he has been on the Faculty of Science and Technology, Keio University. From 1997 to 1999, he joined the Virtualized Reality Project in the Robotics Institute, Carnegie Mellon University as a visiting researcher. Since 2006, he has been a full professor in the Department of Information and Computer Science, Keio University. His recent activities for academic conferences include being Program Chair of ACCV2014, a General Chair of ISMAR2015, and a Program Chair of ISMAR2016. His research interests include computer vision and pattern recognition, and their applications to augmented reality, virtual reality, and human-robotics interaction.

Open Access The articles published in this journal are distributed under the terms of the Creative Commons Attribution 4.0 International License (http:// creativecommons.org/licenses/by/4.0/), which permits unrestricted use, distribution, and reproduction in any medium, provided you give appropriate credit to the original author(s) and the source, provide a link to the Creative Commons license, and indicate if changes were made.

Other papers from this open access journal are available free of charge from http://www.springer.com/journal/41095. To submit a manuscript, please go to https://www. editorialmanager.com/cvmj. 